\title{
A Brief Explanation of the Logic behind the Location Choice of Fast Food Restaurants in China
}

\author{
Muyan Xie \\ International School, Wuxi Big Bridge Academy, Wuxi, China \\ Email: 1756314803@qq.com
}

How to cite this paper: Xie, M. Y. (2021). A Brief Explanation of the Logic behind the Location Choice of Fast Food Restaurants in China. Open Journal of Social Sciences, 9, 466-477.

https://doi.org/10.4236/jss.2021.99034

Received: August 31, 2021

Accepted: September 19, 2021

Published: September 22, 2021

Copyright $\odot 2021$ by author(s) and Scientific Research Publishing Inc. This work is licensed under the Creative Commons Attribution International License (CC BY 4.0).

http://creativecommons.org/licenses/by/4.0/

\section{cc) (i) Open Access}

\begin{abstract}
Chinese market is relative new for fast food chains. In this article, we will explore the competition between the McDonald's and KFC. We will review two papers, each one focusing on one different aspect of the competition mode we are studying. For the Mayer's model, we can apply the profit's concept into our Hotelling model. For the electoral vote model, we can incorporate the concept of market share. We will try to quantify the variables involved in the real world fast food market. We will assume an imaginary line of positions for the firms to choose. Then, in the section of Theoretical Nash Equilibrium, we will try to solve the strategic setting and apply the principles further in the extensions. Finally we will find empirical evidence, which conforms perfectly to our propositions. To sum up, we propose that KFC and McDonald's clustering at the center are of reason, generating maximum profit and market share, creating a stable Nash Equilibrium.
\end{abstract}

\section{Keywords}

Hotelling's Model, Cournot Competition, Location Choice, Market Share, Profit Maximization

\section{Introduction}

Fortuitously, the other day I was walking on the street, wondering about a place to eat. I walked into the mall. The first thing that I notice is that McDonald's and KFC were opening up their stores right next to each other.

In classical city arrangement plans, there are always restaurants designated to cluster within a single mall. Most people do not question the rationality behind the phenomenon. However, based on established theories, all restaurants, which 
in this case, the firms, are supposed to be profit seekers and they are intending to maximize their net benefits all the time.

One of the most important factors in determining the net gain of the firms is the quantity they sold. As long as marginal cost is still under the marginal benefits, the firms should keep producing and try to sell all the products. This leads us to suppose that one vital factor in the competitive markets should be the number of costumers the firm can attract in the location they chose.

In common sense, it seems that clustering in a single place is unusual. This is supposed to decrease the number of consumers into the firm each day because of the competition. The firms should earn more by spreading out and monopolizing over a single district.

In this article, we will check the logic behind this phenomenon.

In statistical aspects, the number of fast food restaurants in China is almost exponentially exploding as can be manifested by the growth of KFC in China alone, indicating that the trend of cluster in the industry is actually worth investigation, as the growth of KFC, for instance, has been a perfect example, with approximately annual growth rate of $8 \%$ in China.

Chinese market in Food and related consumption summed up to account for $4.7 \%$ of the gross domestic product. Thus, understanding the competition pattern in it is fairly important.

As we are inspired by the fast restaurants, we will investigate the competition between them in the article. KFC possesses most of the market share in fast food in China while McDonald's is its most competitor over the market. The logic behind the seemingly irrational location choosing between the two formidable oligopolies will be complex.

And below, we will apply the central concepts of Hotelling's Model in our paper. We will first examine previous literature in this area. Then, we will build up our own mathematical model to explain the logic in the Model Section, presenting mathematical inference in the Results and Discussion Section. The overall summary will be provided in the last part, Conclusion Section.

\section{Literature Review}

We can find some previous literatures that are of high value towards our research. We will account for the strategic setting and Nash Equilibrium set proposed in each model now.

Spatial Cournot competition and heterogeneous production costs across locations by Mayer

One of the most notable ones is the Spatial Cournot competition and heterogeneous production costs across locations (Thierry Mayer, 2000), which describes the situation in which two duopoly firms are competing while trying to maximize their profit by choosing a location in a city.

In this journey, the author considers the situation involving the transportation cost and non-uniform production distribution. The inclusion of transportation 
made this paper quite unique in settings and the final conclusions.

For proposition 1, the author concludes that if the production cost is uniform across the line, the Nash Equilibrium point is when firm 1 and 2 both locate at $1 / 2$, where $l$ is the line that the firm can choose to locate at.

The calculations for this conclusion are pretty complex. The profit earned at each single point, $Z$, should be influenced by the location chosen by both firm 1 and firm 2, where $t$ is the transportation cost, $Q$ is the total quantity produced by the two firms and $q$ is the individual output of the firm:

$$
P_{1}\left(z ; z_{1}, z_{2}\right)=\left[1-Q\left(z ; z_{1}, z_{2}\right)-t\left|z-z_{1}\right|-c\left(z_{1}\right)\right] q_{1}\left(z ; z_{1}, z_{2}\right) \text {. }
$$

If we integrate the profit at each location, we will get the following overall profit of the firm:

$$
P_{1}\left(z_{1}, z_{2}\right)=\int_{0}^{1} \pi_{1}\left(z ; z_{1}, z_{2}\right) \mathrm{d} z
$$

For the firms to maximize their profit, the following conditions need to be met:

$$
\frac{\partial P_{1}}{\partial z_{1}}=0, \frac{\partial^{2} P_{1}}{\partial z_{1}^{2}}<0
$$

If we assume that firm 2 choose $1 / 2$ first, then the profit maximizing point of firm 1 can be solved using the following equations:

$$
\frac{9}{4 t} \frac{\partial P_{1}}{\partial z_{1}} \text {, when } z_{2}=l / 2=A \cdot c^{\prime}\left(z_{1}\right)+B \cdot\left(l-2 z_{1}\right)=0
$$

where

$$
A=\left[-l\left(\frac{1-2 c\left(z_{1}\right)+c(l / 2)}{t}+2 z_{1}-\frac{3}{4} l\right)+2 z_{1}^{2}\right]
$$

and

$$
B=\left[\left(1-2 c\left(z_{1}\right)+c(l / 2)\right)-\frac{t}{4}\left(3 l+2 z_{1}\right)\right]
$$

By solving the expression, the author got that

$$
\left(l-2 z_{1}\right)\left[1-c-\frac{t}{4}\left(3 l+2 z_{1}\right)\right]=0
$$

Using this expression, we can get the unique equilibrium point at $1 / 2$.

The setting of this sub model is similar to the previous one. There are still two firms competing to maximize their profit, but the proposition under the assumption that the production cost is not uniform can be vastly different from the one stated before.

The author proposed the following conclusion:

The most useful one is that "for non-uniform production cost distributions, central agglomeration can only be sustained if $1 / 2$ is the locally cost minimizing location. If the production cost distribution is globally convex with $1 / 2$ yielding the minimum production cost, central agglomeration is the unique equilibrium." 
We need to resolve the differences in the two models or at least justify that the differences have minor effect to ensure that we can use formulas and assumptions from the literature.

The biggest concern we need to address here is the relevance of transportation costs in the models. In the Mayer's paper, he introduced the concept of non-uniform cost function, which is of huge influence on the final results and conclusions of the paper. The production, as he assumed, may be non uniform along the line in two parts, one being the transportation costs being non linear and the other being the rent being non uniform.

However, in our study of the competition between McDonald's and KFC, transportation cost can even be neglected. The main contributor in the transportation cost, as the article may refer, is the cost needed to bring the product from the firm to the costumer. For these two fast food restaurants, takeout only counts for little of the income, with most people eating at the site of the restaurant.

However, we can discern from Equations (1) to (4), transportation cost does not appear in the decision determinant equations. Thus it is suitable for us to disregard the effect of transportation cost in both situations.

Eliminating this difference, we can judge that the propositions and logic from this paper is good for us to use now.

The Hotelling's model of electoral competition from the Osborne book

The other journal worth noticing is the Hotelling's model of electoral competition in the book The Osborne.

In this section, the author explains the application of the Hotelling's model in the elections. In the game, each candidate will express their own opinion on a certain matter and will choose a location on a line if we categorize positions toward a political topic onto a continuous line.

The players in the game, which in this case are the candidates, are stick to the belief that they need to choose a position in order to win the most favor from the public. Each person will non-uniformly stand to a point on the line. They will choose to vote for the candidate that reflect, or support, their own position the best.

The settings in the game of election conform to our setting in our analysis of the game in fast food restaurants. There are two players and each player is trying to maximize their payoff function.

$$
B_{1}\left(x_{2}\right)=\left\{\begin{array}{l}
\left\{x_{1}: x_{2}<x_{1}<2 m-x_{2}\right\}, x_{2}<m \\
\{m\}, x_{2}=m \\
\left\{x_{1}: 2 m-x_{2}<x_{1}<x_{2}\right\}, x_{2}>m
\end{array}\right.
$$

In the scenario above, $x_{1}$ and $x_{2}$ are the locations of the candidate. The payoff for candidate 1 depends on its own location and, at the same time, the location chose by candidate $2 . m$ is the mid of the line of positions.

Candidate 2 faces the same scenario as candidate 1 . They two have exactly the same best response function. If we superimpose the two functions, we can get a 
unique Nash Equilibrium solution set. It is when the two candidates choose $\mathrm{m}$ at the same time, each time attracting half of the voters.

The main difference we need to be aware of here is that winning the most votes is not parallel to earning the maximum profit.

Profit maximization involves balancing between cost, sales and price while winning the most votes can be compared to winning the biggest market share. Each voter is like a consumer looking for products. Attracting the most consumers for shopping in the specific firm is the same thing as winning the favor of the votes from the voters. We define market share in Economics to be sharing of the purchases in the overall industry, making it parallel to attracting votes.

With this in mind, we can try to give out models based on market share maximization using the conclusion above.

\section{Methods}

Now we will try to develop our own unique strategic settings for the model, we will propose our assumptions and justifications below.

\section{Assumptions}

First of all, we will assume that there are only two firms, McDonald's and KFC, competing for profit in the local areas. Judged from the previous data, McDonald's and KFC in total share almost $60 \%$ of the fast food restaurant share in China, thus it is fine for use to use $\mathrm{M}$ and $\mathrm{K}$ to represent the two firms we are studying.

Secondly, we may assume that the only actions the firms can take are choosing the location to open their shops and choose the quantity they wish to sell everyday (Abhijit Sengupta \& Kunal Sengupta, 2008). It is quite simple that each firm is able to choose freely the location to offer the service. Also, in Chinese Market, these two firms are truly forming the duopoly. They have the power to choose the quantity for sell by setting the numbers they cook everyday. They will need to make decisions simultaneously.

Next, we may assume that originated from Capitalism, the two firms are profit seekers and they are always trying to maximize their profit.

Also, consumers in China should be totally unfamiliar with the western style foods. They will treat the products as absolutely identical. They will select the firm to consume based solely on the distance between themselves and the restaurant.

Then, we assume temporarily that the production cost at each site is the same. Finally and the most crucial part is the payoff function of the firms. On the imagined line of location, we can assume that the two identical firm, one being fm, being McDonald's and the other being $f_{k}$, representing KFC, with their corresponding place chosen as $z_{m}$ and $z_{k}$. It is reasonable that we assume the line of length $l$ and $z_{m}$ is to the left of $z_{k}$.

We will get the following equations for payoff for $f_{m}$ and $f_{k}$ :

$$
P_{m}=\int_{0}^{\frac{z_{m}+z_{k}}{2}}\left(\frac{a-c}{3}\right)^{2} \mathrm{~d} l=\left(\frac{a-c}{3}\right)^{2}\left(\frac{z_{m}+z_{k}}{2}\right)
$$


and

$$
P_{k}=\int_{0}^{l-\frac{z_{m}+z_{k}}{2}}\left(\frac{a-c}{3}\right)^{2} \mathrm{~d} l=\left(\frac{a-c}{3}\right)^{2}\left(l-\frac{z_{m}+z_{k}}{2}\right)
$$

We will present the mathematical process that leads us toward the payoff function in the subsection below.

\section{Justifications based on Math}

We hold the below truths to be self evident, that the two firms are engaged in Cournot Competition, in which each firm has the option to compete using quantity. The conditions for the Cournot competition to occur are satisfied.

We can express the profit the firm earns at a single point as the following equation, as we use $c$ to represent the cost function:

$$
\pi_{m}=p_{m} q_{m}-c q_{m}, \pi_{k}=p_{k} q_{k}-c q_{k}
$$

The $p_{m}$, or the price of the products in firm $m$, can be expressed as $\alpha-q_{m}-q_{k}$ with $\alpha$ being a constant, indicating that the price is influenced by the output sum of the two firms.

Then we can transform Equation (9) to the following forms:

$$
\pi_{m}=\left(\alpha-q_{m}-q_{k}\right) q_{m}-c q_{m}=\left(\alpha-q_{m}-q_{k}-c\right) q_{m}
$$

And

$$
\pi_{k}=\left(\alpha-q_{m}-q_{k}\right) q_{k}-c q_{k}=\left(\alpha-q_{m}-q_{k}-c\right) q_{k}
$$

As each firm can only maximize their profit at the point if they satisfy the following condition:

$$
\frac{\partial \pi_{m}\left(q_{m}, q_{k}\right)}{\partial q_{m}}=0=\alpha-2 q_{m}-q_{k}-c
$$

Similarly, by solving the condition for firm $k$ we can get that $\alpha-2 q_{k}-q_{m}-c$ $=0$.

Combining the two equations, we can get the conclusion that:

$$
q_{m}=q_{k}=\frac{a-c}{3}
$$

The overall payoff at a single point will be:

$$
\pi_{m}=p_{m} q_{m}-c q_{m}=\left(\frac{a+2 c}{3}\right)\left(\frac{a-c}{3}\right)-c\left(\frac{a-c}{3}\right)=\left(\frac{a-c}{3}\right)^{2}
$$

The overall profit will simply be the integration:

$$
P_{m}=\int_{0}^{\frac{z_{m}+z_{k}}{2}}\left(\frac{a-c}{3}\right)^{2} \mathrm{~d} l=\left(\frac{a-c}{3}\right)^{2}\left(\frac{z_{m}+z_{k}}{2}\right)
$$

\section{Results and Discussion}

In this section, we will try to propose the Nash Equilibrium for the duopoly competition in fast food market.

Gripping Equation (14), we can see that firm $\mathrm{m}$ has full incentive to move to- 
ward right. Judging from Equation (7), we can see that firm $k$ has good motivation to move to the left.

They will keep doing so until they meet each other. We will designate the place they met as $Z_{x}$.

The varying parts in Equations (6) and (7) are $\frac{z_{m}+z_{k}}{2}$ and $\frac{2 l-z_{m}-z_{k}}{x}$, respectively.

After they met, the determinant part will be $z_{x}$ and $\frac{2 l-z_{k}}{2}$. The game will be in Nash Equilibrium only when the two parts are equal in value, making the two firms earn the same amount, indicating that $z_{x}$ should be at $1 / 2$.

Proposition 1:

Under the assumption that the firms are profit seekers and that the distribution of production cost is uniform along the line and without consideration of the rent, the unique Nash Equilibrium exists at the center of the line with two players, in this case, McDonald's and KFC, clustering next to each other.

But there is a problem here. The production cost may not always be uniform and they will need to conform to some distribution.

To be specific, the production cost involves in producing the fast food can be classified as unit specific cost and fixed costs. For unit specific cost consists of the cost of the raw material, which we will assume to be unchanging for each unit.

The next part is the fixed costs. These costs can be influenced by the location chosen by the firm. A large part of the fixed costs is the rent. In common sense, the rent should be higher for places near the city center and lower in the suburbs, meaning that we can use the following chart to define the rent cost function of the firms in Figure 1.

In Figure 1, the quadratic function is used to depict the common rent rate, with climax at point $(I / 2, \theta)$ and basic price of rent of $\beta$ at the start or the end of the line.

We can use the following function to describe the quadratic distribution:

$$
R_{z}=\frac{4(\beta-\theta)}{l^{2}} z^{2}+\frac{4(\theta-\beta)}{l} z+\beta
$$

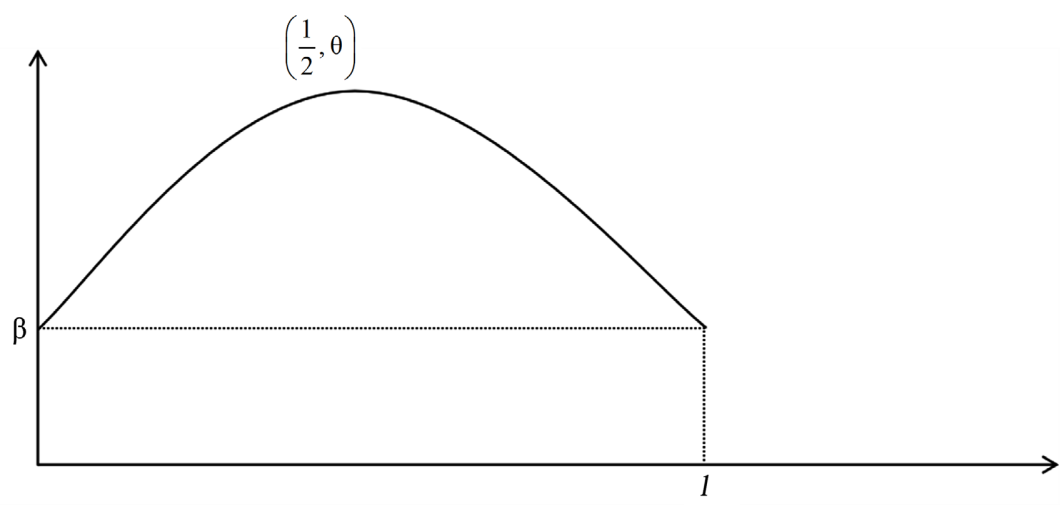

Figure 1. Relation between rent cost and location on the line. 
with $z$ being the exact location of the firm on the line, $\beta$ being the lowest cost which is assumed at the end of the line and $\theta$ being the highest cost at the center of the line.

As we are considering rent here, we must edit our expression for overall profit of the firm in the following ways:

$$
\begin{aligned}
N_{m} & =\left(\frac{a-c}{3}\right)^{2}\left(\frac{z_{m}+z_{k}}{2}\right)-R_{z_{m}} \\
& =\left(\frac{a-c}{3}\right)^{2}\left(\frac{z_{m}+z_{k}}{2}\right)-\frac{4(\beta-\theta)}{l^{2}} z_{m}^{2}-\frac{4(\theta-\beta)}{l} z_{m}-\beta
\end{aligned}
$$

And

$$
\begin{aligned}
N_{m} & =\left(\frac{a-c}{3}\right)^{2}\left(\frac{2 l-z_{m}-z_{k}}{2}\right)-R_{z_{k}} \\
& =\left(\frac{a-c}{3}\right)^{2}\left(\frac{2 l-z_{m}-z_{k}}{2}\right)-\frac{4(\beta-\theta)}{l^{2}} z_{k}^{2}-\frac{4(\theta-\beta)}{l} z_{k}-\beta
\end{aligned}
$$

with $N$ representing the net profit of the firm.

In order to get the maximum profit, we need to satisfy the following equations:

$$
\begin{gathered}
\frac{\partial N_{m}\left(z_{m}, z_{k}\right)}{\partial z_{m}}=0=\frac{1}{2}\left(\frac{a-c}{3}\right)^{2}-\frac{8(\beta-\theta)}{l^{2}} z_{m}-\frac{4(\theta-\beta)}{l} \\
\frac{\partial^{2} N_{m}\left(z_{m}, z_{k}\right)}{\partial z_{m}^{2}}=-\frac{8(\theta-\beta)}{l^{2}}<0
\end{gathered}
$$

And

$$
\begin{gathered}
\frac{\partial N_{k}\left(z_{m}, z_{k}\right)}{\partial z_{k}}=0=-\frac{1}{2}\left(\frac{a-c}{3}\right)^{2}-\frac{8(\beta-\theta)}{l^{2}} z_{k}-\frac{4(\theta-\beta)}{l} \\
\frac{\partial^{2} N_{m}\left(z_{m}, z_{k}\right)}{\partial z_{k}^{2}}=-\frac{8(\theta-\beta)}{l^{2}}<0
\end{gathered}
$$

By solving Equations (18) and (20), we get that:

$$
z_{m}=\frac{l}{2}+\frac{l^{2}}{16}\left(\frac{a-c}{3}\right)^{2}=\frac{l}{2}+\frac{l^{2}}{16} \pi_{m}
$$

And

$$
z_{k}=\frac{l}{2}-\frac{l^{2}}{16}\left(\frac{a-c}{3}\right)^{2}=\frac{l}{2}-\frac{l^{2}}{16} \pi_{k}
$$

The apparent problem in this solution is that the precondition for the equations is that $z_{m}$ is to the left of $z_{k}$, which is contradictory in this case.

Also, as we are checking the second order conditions, as in Equations (19) and (21), we see that $\beta-\theta$ is less than 0 , meaning that the expression $-\frac{8(\beta-\theta)}{l^{2}}$ should forever be bigger than 0 , meaning the second order condition will never be satisfied. 
Here we can conclude that mathematically we can never fetch a solution for these equations.

Thus we should resort to programming to verify the Nash Equilibrium.

In the program, we made the two firm initiate at the two ends of the line. They can simultaneous make decision to adjust their position to larger their position. We will use Monte Carlo simulation method (Owen, 2009) to ensure that the step is small enough that true strategic situation can be captured.

After running the code, we can see that the Nash Equilibrium arrives exactly at the mid of the value of the line of points possible to locate the firm. Thus we may come to proposition 2 .

Proposition 2:

If we introduce the concept of non uniform production cost, which, in the competition between the fast food restaurants, should be the non uniform rent distribution along the line, we will find out that the two firm will still aggregate at the mid of the line

\section{Extensions}

Now we will try to extend our models to some other situations.

McDonald's and KFC are new to Chinese consumers. At the beginning, they will definitely tend to focus on the maximization of market share in order to enlarge their market influence and win edge over the competitors.

As we have ruled before, the model we established focused on the profit in the Cournot Competition.

We have already justified ourselves in the applicability of the model of electoral vote attraction in the competition between the two fast food restaurant if they are overwhelmed with the idea of attracting the most consumers.

Thus, applying exactly the same principle and logic in the electoral voting model, we can get the following proposition:

Proposition 3:

Under the situation that the two firms are competing for the largest market share, the Nash Equilibrium will be at the midpoint of the line, with both firms agglomerating and both sharing half of the market.

Now we can add one player into the game: Hamburger King. It is also competing with McDonald's and KFC to take up the most market in China.

The payoff function for the situation should be pretty complex. If the three firms choose the same location, then they should each share $1 / 3$ of the market. If all three are clustering at the center and anyone may choose to move, its market share will instantly decrease, meaning that all clustering center can be a Nash Equilibrium.

If two firms choose the same location, the payoff will be different. If we assume that the firm from the left to the right to be $f_{1}, f_{2}$ and $f_{3}$. Thus we can get that $Z_{1}=Z_{2}<Z_{3}$ or $Z_{1}<Z_{2}=Z_{3}$.

The functions in Figure 2 describe the payoff function under either situation.

For the left function, we can see that if $\mathrm{xx}$ moves its location to the left, it can 


$$
\left\{\begin{array}{c}
P\left(x_{1}\right)=\frac{x_{1}+x_{3}}{4} \\
P\left(x_{2}\right)=\frac{x_{1}+x_{3}}{4} \text { or }\left\{\begin{array}{c}
P\left(x_{1}\right)=\frac{x_{1}+x_{3}}{2} \\
P\left(x_{2}\right)=\frac{2 l-x_{1}-x_{3}}{4} \\
P\left(x_{3}\right)=\frac{2 l-x_{1}-x_{3}}{2}
\end{array} \text { ( } x_{3}\right)=\frac{2 l-x_{1}-x_{3}}{4}
\end{array}\right.
$$

Figure 2. Relation between rent cost and location on the line.

earn more market share. On the right, if $x_{1}$ move itself to the right, it can win more shares. There is no Nash Equilibrium.

Then if the three firms each choose a different location, we can assume $Z_{1}<Z_{2}$ $<z_{3}$. Thus we can the following payoff function:

$$
\left\{\begin{array}{l}
P\left(x_{1}\right)=\frac{1}{2}\left(x_{1}+x_{2}\right) \\
P\left(x_{2}\right)=\frac{1}{2}\left(x_{3}-x_{1}\right) \\
P\left(x_{3}\right)=l-\frac{x_{2}+x_{3}}{2}
\end{array}\right.
$$

Let's go through the logic here. $x_{2}$ cannot determine its own payoff. So we can focus on $x_{1}$ first. It can maximize its profit as it is getting closer to $x_{2}$. On the other hand, $x_{3}$ can maximize its profit as it gets closer to $x_{2}$.

If the three firms are all allowed to move, they will finally converge to one point. The electoral vote model proposes that there should be no Nash Equilibrium. The only difference that divert the conclusion from ours is that each one sharing $1 / 3$ of the poll is not satisfying for them. They would rather to move than tie.

Proposition 4:

Assuming all other conditions and assumption same as in the previous proposition, the 3 player game will at least have a Nash Equilibrium at the center as the three clustering there, each sharing equal market

\section{Empirical Evidence}

Though the competition mode between KFC and McDonald's are intense and attract loads of researches in the area, no people have considered the competition mode in location choosing perspective and Hotelling model's application.

Thus, we need to find the data by ourselves. Below we will show the illustration generated from Google Maps in Figure 3.

In the figure, we pinpoint the location of KFC in a city with a star mark and the location of McDonald's with a fork mask. Judging from the figure, we can see that our conclusions that under all circumstances, either for uniform or non uniform production cost distribution, the two duopoly will cluster at the center of the city in order to maximize their profit or their market share.

Upon reviewing a paper published by Presh Talkwalkar at Stanford University Mathematical Economics Major (Presh Talwalkari et al., 2012), I find some useful data on the location of McDonald's and Hamburger King in the United States. Though at a different location and studying different firms, I find the conclusions of high value. 


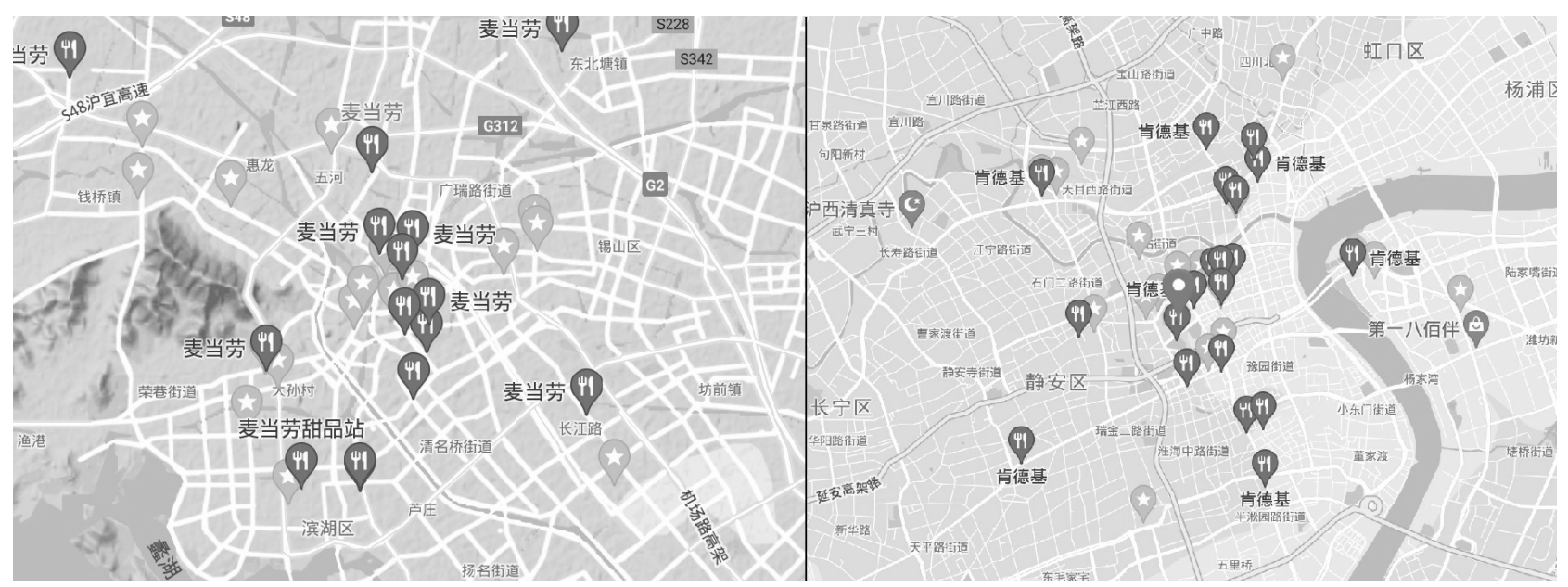

Figure 3. Location of KFC and McDonald's in the city of Wuxi (Left) and Shanghai (Right). ${ }^{*}$ Please notice that there are two identifiers in the charts above. The one with a folk and a knife stands for either KFC or McDonald's and the one with the star mark stands for the other restaurant.

He focused intensely on the competition over the market share instead of the profit and treat the game sequentially, as he assume that firms can pop up chain stores in the same city.

He concluded that all firms should cluster at the center initially and spread its influence all over the city by expanding along the line, making our propositions similar to his. He gets empirical support from the website of fastfoodmaps.com, locating the firms' location in each state in US. The overall data is pretty complex and we can review them only on the website.

To sum up, empirically, Chinese data and US papers conforms to the propositions we promote here in the paper.

\section{Conclusion}

While the models are trying to be perfect, limitations still exist. First of all, the modelling of rent prices could be concluded using regression models of empirical data, which due to restriction of time and resources, was not conducted in the research. Second, more firms may compete in the new market. If we wish to extend the applicability from fast food industry, we may need to consider 4 or 5 player games in the models.

The good thing is that the result and propositions obtained through the mathematical models are fairly simple. Whether the duopoly is considering maximizing their profit or market share, they will have a Nash Equilibrium at the center of the line.

If there are three players competing for market share, clustering at the center of the line and equally sharing the consumer pool is one Nash Equilibrium.

These propositions can help us to better understand the strategies the western food restaurants can use when they are trying to explore new markets. In the new markets, they are treated almost as identical, offering undifferentiated products. To ensure their own success, they should choose to locate their store at the 
center of the line of positions.

Thus, it is fairly simple for us to conclude that McDonald's and KFC are following the pattern and perform according to their best interest.

\section{Acknowledgements}

Special thanks here toward my professor Mark Foley. This article can never be possible without his sincere help. It is him who inspired me and gave me the determination to study economics lifelong.

I am extremely grateful for those who give their best help to me in pursuing my academical career. I cannot stand here without their sincere aid.

\section{Conflicts of Interest}

The author declares no conflicts of interest regarding the publication of this paper.

\section{References}

Mayer, T. (2000). Spatial Cournot Competition and Heterogeneous Production Costs across Locations. Regional Science and Urban Economics, 30, 325-352. https://doi.org/10.1016/S0166-0462(99)00043-5

Owen, A. B. (2009). Monte Carlo and Quasi-Monte Carlo for Statistics. In P. L'Ecuyer, \& A. B. Owen (Eds.), Monte Carlo and Quasi-Monte Carlo Methods 2008 (pp. 3-18), Springer. https://doi.org/10.1007/978-3-642-04107-5 1

Sengupta, A., \& Sengupta, K. (2008). A Hotelling-Downs Model of Electoral Competition with the Option to Quit. Games and Economic Behavior, 62, 661-674.

https://doi.org/10.1016/j.geb.2007.06.008

Talwalkari, P. et al. (2012). Why Are McDonald's and Burger King Usually Located near Each Other? Fast Food Location Game Theory. Mind Your Decisions.

http://mindyourdecisions.com/blog/2012/10/23/why-are-mcdonalds-and-burger-kingusually-located-near-each-other-fast-food-location-game-theory/ 